\title{
LUMBAR CANAL STENOSIS: THE SYNDROMES AND THEIR MANAGEMENT
}

\author{
IAN P. CAST, M.B., B.S., F.R.C.S., F.R.C.S.(Ed.) \\ Consultant Neurological Surgeon, Department of Surgical Neurology, \\ Morriston Hospital, Swansea, SA6 6NL
}

An account is given of the syndromes associated with lumbar spinal stenosis and their surgical management. One hundred and ten patients were treated by two neurosurgeons in a sub-Regional Department of Surgical Neurology over a Io-year period, and an analysis was carried out. In the series described, men outnumbered women by 2 to I, in contrast to the closer I to I sex distribution of simple lumbar disc lesions. Age at presentation tends to be later than in disc lesions. Thirty-four patients gave histories extending back for to years or more.

The characteristic syndrome of lumbar spinal stenosis is neurogenic claudication. However, when developmental stenosis is associated with degenerative changes in the spine or disc prolapse, bilateral sciatica and cauda equina syndromes are more usual.

Stenosis of the lumbar canal may be unisegmental, or, more usually, multisegmental; unilateral or bilateral. It is frequently associated with developmental cervical stenosis and complicated by disc protrusion and arthritic states. Minor trauma may be a presenting factor.

Most forms of stenosis can be diagnosed from plain X-ray studies. More specialised investigation is mandatory for the proper appraisal of the extent of the condition, and so that adequate surgical management may be planned.

A good result from surgical treatment depends upon wide bony decompression, often including removal of both the inferior and the superior articular facets, at all affected levels. If there are continuing or relapsing symptoms, the surgeon must be prepared to re-operate and extend the decompression. Despite the often necessary extensive removal of bone, spinal instability has not proved to be a significant complication in our series. All three patients with post-operative instability were patients who had rheumatoid arthritis.

The best results were obtained in uncomplicated developmental stenosis, but some improvement was obtained in all of the survivors; there was only one death.

* This is a Summary of a paper which was read at the I.M.S.P. Meeting, Stoke Mandeville, England, July, I98I. 